\title{
Brain amyloid in virally suppressed HIV-associated neurocognitive disorder
}

Gemma C. Howdle, BA\&Sc, Yann Quidé, PhD, Mustafa S. Kassem, PhD, Kate Johnson, MD, Caroline D. Rae, PhD, Bruce J. Brew, MD, DSc, FRACP, FAAN, and Lucette A. Cysique, PhD

Neurol Neuroimmunol Neuroinflamm 2020;7:e739. doi:10.1212/NXI.0000000000000739

\section{Abstract}

\section{Objective}

To determine whether virally suppressed HIV neuropathogenesis, a chronic neuroinflammatory state, promotes abnormal brain amyloid deposition.

\section{Methods}

A total of 10 men with virally suppressed HIV-associated neurocognitive disorder (HAND), aged 46-68 years, underwent ${ }^{11}$ C-labeled Pittsburgh compound B PET. Data from the Australian Imaging, Biomarkers and Lifestyle (AIBL), including 39 cognitively normal individuals (aged 60-74 years), 7 individuals with mild cognitive impairment (MCI) (aged 64-71 years), and 11 individuals with Alzheimer disease (AD) (aged 55-74 years), were used as reference. Apart from more women, the AIBL cohort was demographically comparable with the HIV sample. Also, the AIBL PET data did not differ by sex. Cerebellum standardized uptake value ratio amyloid values within 22 regions of interest were estimated. In the HIV sample, apolipoprotein E (APOE) was available in $80 \%$, CSF biomarkers in $60 \%$, and $8-10$ years of long-term health outcomes in $100 \%$.

\section{Results}

HAND and the AIBL group with no cognitive deficits had similar amyloid deposition, which was lower than that in both the $\mathrm{MCI}$ and $\mathrm{AD}$ groups. At the individual level, one HAND case showed high amyloid deposition consistent with $\mathrm{AD}$. This case also had a CSF-AD-like profile and an E4/E4 for APOE. Clinically, this case declined over 18 years with mild HAND symptoms first, followed by progressive memory decline 8-9 years after the study PET, then progression to severe dementia within 2-3 years, and lived a further 6 years. Another HAND case showed increased amyloid deposition restricted to the hippocampi. Two other HAND cases showed abnormally decreased amyloid in subcortical areas.

\section{Conclusions}

Relative to cognitively normal older controls, brain amyloid burden does not differ in virally suppressed HAND at the group level. However, individual analyses show that abnormally high and low amyloid burden occur.

\author{
Correspondence \\ Dr. Cysique \\ Icysique@unsw.edu.au
}




\section{Glossary}

AAL = Automated Anatomical Labeling; $\mathbf{A} \beta=$ amyloid beta; $\mathbf{A D}=$ Alzheimer disease AIBL $=$ Australian Imaging, Biomarkers and Lifestyle; ANI = asymptomatic neurocognitive impairment; APOE = apolipoprotein E; ${ }^{11} \mathrm{C}-\mathrm{PiB}={ }^{11} \mathrm{C}$-labeled Pittsburgh compound $\mathrm{B} ;$ cART $=$ combination antiretroviral therapy; HAD $=$ HIV-associated dementia; HAND $=$ HIV-associated neurocognitive disorder; $\mathbf{M C I}=$ mild cognitive impairment; $\mathbf{M N D}=$ mild neurocognitive disorder; $\mathbf{M S K}=$ Memorial Sloan Kettering; NNTC $=$ National NeuroAIDS Tissue Consortium; PLHIV = people living with HIV infection; PSP $=$ progressive supranuclear palsy; PVE = partial volume effect; ROI = region of interest; SUVR = standardized uptake value ratio.

The neuropathogenesis of HIV is commonly characterized by chronic neuroinflammation in people living with HIV infection (PLHIV) who are aging and virally suppressed on combination antiretroviral treatment (cART). ${ }^{1}$ Both age and HIV-associated chronic inflammation may be associated with a higher risk of developing neurodegenerative disease such as Alzheimer disease (AD), ${ }^{1}$ especially as PLHIV are entering age where dementia starts to rise in the general population. ${ }^{2}$ $\mathrm{AD}$ and other forms of dementia and predementia syndromes are often characterized by abnormal deposition of cortical amyloid. $^{3,4}$ This neuropathologic process may be used as a way to identify aging PLHIV at risk of dementia and may represent one of the endpoints of HIV-related chronic neuroinflammation. One of the most efficient detection methods for brain amyloid deposition in the living brain is the $\beta$-amyloid radioligand ${ }^{11} \mathrm{C}$-labeled Pittsburgh compound B $\left({ }^{11} \mathrm{C}\right.$ $\mathrm{PiB})$ positron emission tomography (PET). ${ }^{5}$ To date, there is no clear evidence that middle-aged PLHIV are more at risk of developing $\mathrm{AD}$ than their HIV-negative (HIV-) counterparts. In particular, it remains unclear whether HIV leads to $\mathrm{AD}$ or to an $\mathrm{AD}$-like neurodegeneration for the following reasons.

Evidence from the literature remains limited and inconsistent. Achim et al. $^{6}$ studied 40-65-year-old HIV+ participants using the molecular imaging probe $2-(1-\{6-[(2-[\mathrm{F}-18]$ fluoroethyl)(methyl) amino]-2-naphthyl\} ethylidene)malononitrile (FDDNP)-PET and found increased binding of $\beta$-amyloid and tau protein aggregates in the posterior cingulate gyrus and the parietotemporal areas in three cases with mild neurocognitive disorder (MND). Furthermore, immunohistochemistry of the neocortex, subcortical white matter, and basal ganglia in HIV+ patients on cART found that 34 of 35 of these cases had intraneuronal deposition of $\beta$-amyloid. Tau and $\alpha$-synuclein were also abundant with the distribution following axonal tracts. APOE genotyping, the major genetic $\mathrm{AD}$ risk factor, was not performed, and there were no concomitant neurocognitive data. Another study by Ances et al. ${ }^{7}$ that included 10 cognitively unimpaired $\mathrm{HIV}+$ individuals (mean age 52 years, age range $46-58$ years, $50 \%$ men) did not show an overall increased amyloid deposition using ${ }^{11} \mathrm{C}-\mathrm{PiB} / \mathrm{PET}$ compared with $20 \mathrm{HIV}-$ controls (age range $41-53$ years, $80 \%$ men). This was despite 4 of 10 participants having low CSF amyloid beta (A $\beta) 42$ levels. The same research group led by Ances et al. ${ }^{8}$ also found no significant evidence of pathologic amyloid deposition with ${ }^{11} \mathrm{C}-\mathrm{PiB} / \mathrm{PET}$ when compared with HIV- community age-comparable controls $(88 \%$ men) in $5 \mathrm{HIV}$-associated neurocognitive disorder
(HAND) cases (82\% men) and 11 cognitively unimpaired HIV+ patients (100\% men) aged in their 40s. The HIV+ groups had a significantly lower amyloid deposition than $11 \mathrm{AD}$ cases aged in their $70 \mathrm{~s}$ ( $55 \%$ men). APOE genotyping was conducted in $>90 \%$ of the HIV + participants, and $45 \%-50 \%$ has at least one APOE 4 allele, but this was not associated with increased amyloid deposition. Analyses of CSF-AD markers were conducted in $77 \%$ of the HIV+ sample, with $5 \mathrm{HIV}+$ participants found to have low CSF-A $\beta 42$ levels $(<500 \mathrm{pg} / \mathrm{mL})$. CSF-A $\beta 42$ levels were not found to be associated with increased amyloid deposition. A larger study of $26 \mathrm{HIV}+$ adults aged 26-67 years old, reported as part of a review, found no difference in ${ }^{11} \mathrm{C}$-PiB PET amyloid deposition and CSF-A $\beta 42$ levels between the HIV+ and the HIV- patients aged 32-62 years old. ${ }^{9}$ This study did not provide APOE genotyping, and levels of cognitive performance were not reported. Finally, a case study of an HIV-infected (HIV+) 71-year-old man with an undetectable plasma viral load found a combined diagnosis of HAND and probable $\mathrm{AD} .{ }^{10}$ This was based on a multidisciplinary examination including neuropsychological testing, brain imaging, CSF-AD markers, fluorodeoxyglucose PET, and florbetaben PET.

Overall, ${ }^{11} \mathrm{C}$-PiB PET was the most commonly used method for detecting brain amyloid deposition. All studies conducted have focused on middle-aged PLHIV, mostly without HAND. All studies but one ${ }^{9}$ reported that most patients were on cART, although the degree of viral suppression was variable or not clearly reported. This represents a major caveat because detectable viral load has been found to be associated with increased $\mathrm{A} \beta$-plaque in the hippocampus in a recent National NeuroAIDS Tissue Consortium (NNTC) analysis. ${ }^{11}$ APOE genotyping was conducted in only one study. ${ }^{8}$ While APOE4 status has been associated with HAND in some studies ${ }^{12,13}$ this has not always been replicated, ${ }^{14}$ although this result may be influenced by cohorts that included PLHIV of relatively young age, who were not virally suppressed and clinically stable. ${ }^{14}$ Nevertheless, APOE4 status is a major risk factor for $\mathrm{AD}$ and other dementias ${ }^{14}$ and, thus, remains relevant in aging PLHIV cohorts that could be at higher risk of coincidental $\mathrm{AD}$. Also, APOE4 is a strong modulator of ${ }^{11} \mathrm{C}-\mathrm{PiB}$ PET data. In a cohort of 497 cognitively normal (NL) middle- and older aged participants with ${ }^{11} \mathrm{C}$-PiB PET, ${ }^{15}$ a greater proportion of APOE4 carriers developed amyloid- $\beta$ pathology, at an earlier age, and with faster amyloid- $\beta$ accumulation. Furthermore, a recent and large neuropathologic study from the NNTC showed that APOE4 and age were strong independent 
predictors of the deposition of $4 \mathrm{G} 8$ - $\mathrm{A} \beta$ plaques in the frontal neocortex, evident in $29 \%$ of a selected sample.

${ }^{11} \mathrm{C}-\mathrm{PiB}$ PET has high affinity and selectivity for fibrillar $A \beta$ in plaques and extracellular amyloid deposits. ${ }^{5}$ It may thus lead to lower binding in PLHIV and patients with HAND for whom plaques have been found to be more often diffuse and intracellular. ${ }^{16-18}$ However, these neuropathologic findings were derived from cohorts that included relatively young patients, active AIDS, as well as many without full viral control and sometimes significant alcohol and drug use comorbidity. ${ }^{17-19}$ In addition, the detection of abnormal amyloid deposition in the oldest PLHIV with HAND using ${ }^{11} \mathrm{C}-\mathrm{PiB}$ PET is important, as $\mathrm{AD}$ may be coincidental. On the other hand, the diffuse type of plaques may only reflect the early phase of the disease.

This study aimed to compare brain amyloid deposition quantified by ${ }^{11} \mathrm{C}-\mathrm{PiB}$ PET in 10 formally diagnosed HAND cases who were part of the Australian HIV and Aging Cohort. HAND cases were screened for hepatitis $C$ infection, nonHIV neurologic conditions, alcohol and drug use to minimize non-HIV-related neuroinflammation, and other forms of brain pathology. ${ }^{20}$ These were compared with 39 NL cases, 7 cases with mild cognitive impairment (MCI), and $11 \mathrm{AD}$ cases aged 55-74 years old, all from the Australian Imaging, Biomarkers and Lifestyle (AIBL) cohort. ${ }^{5,21}$ Because of the relatively small sample size and heterogeneous pattern of amyloid deposition in the HIV+ group, we also conducted a series of case analyses. Finally, we collected long-term health outcomes 8-10 years after the end of the PET data collection to determine dementia progression.

\section{Methods}

The University of New South Wales, St. Vincent's Hospital, and Austin Health Human Research Ethics Committees approved this research protocol (HREC/08/096). Informed written consent or the guardian consent when appropriate was obtained from all participants.

\section{Participants}

Ten HIV+ men formally diagnosed with HAND (table 1) were enrolled in a study to quantify brain amyloid deposition using the $\beta$-amyloid radioligand ${ }^{11} \mathrm{C}$-PiB as part of the Australian HIV and Brain Aging Cohort Study between 2009 and 2011. The inclusion/exclusion criteria have been described in detail previously. ${ }^{22}$ All were on cART with undetectable plasma and CSF viral load $(<50$ copies $/ \mathrm{mL})$. Seven of 10 underwent a lumbar puncture and blood tests to determine CSF-AD markers and HIV markers, respectively, and 8 of 10 had APOE genotyping. All HAND cases underwent neuropsychological testing, and a high-resolution T1-weighted MRI scan was obtained for each person; these methods have been reported in detail elsewhere. ${ }^{13,22,23}$ Five cases had mild HAND, including 4 cases with asymptomatic neurocognitive impairment (ANI) and 1 case with MND. The 5 other cases had HIV-associated dementia (HAD) along its clinical spectrum (Memorial Sloan Kettering [MSK] scale ${ }^{24}$ ), including 1 case with mild HAD (MSK1), 2 with moderate HAD (MSK2), and 2 with severe HAD (MSK3). Note that on the MSK scale, MND is classified as MSK1.

\section{AlBL data}

A request was made to access the summed digital imaging and communications in medicine PET AIBL data on August 28, 2018, at aibl.csiro.au/research/support/ and was approved on August 30, 2018. These data were collected by the AIBL study group following a published protocol. ${ }^{25}$ The AIBL study methodology has also been reported previously. ${ }^{5,21}$ AIBL subjects aged between 55 and 74 years with a PET scanning protocol closest to that of the HIV+ sample were retained (that is PET scan with a duration of 1,200 seconds: 57/103). Demographic and clinical characteristics of the retained AIBL cohort are presented in table 1 . The AIBL sample has comparable demographics with the HIV sample except for more women, which is typical of an elderly cohort, because women have a higher survival rate and are more represented in $\mathrm{AD}$ cases $^{21}$; HIV in Australia remains most prevalent in men who have sex with men. ${ }^{22}$ It is important that there was no significant differences between sexes in the AIBL PET data ( $t$ test $p$ values $>0.50$ ). Although HIV infection was not formally diagnosed in those controls, the AIBL exclusion criteria were stringent and included a wide range of neurologic, psychiatric, and medical conditions in addition to laboratory test abnormalities. ${ }^{5,21}$ Evidence of immune compromise would therefore have been detected. Furthermore, the age and demographics of this cohort limits the risk of HIV infection because older age and late diagnosis are more common in non-Australian-born people. ${ }^{26}$ See examples of AIBL PET data in figure e-1 and e-3, links.lww. com/NXI/A248, in supplemental material.

\section{PET data acquisition in the HIV+ sample}

All PET scanning took place at the Austin Health PET Centre in Melbourne (VIC, Australia). Patients were flown from Sydney and back, with their carer as appropriate. They were requested to fast for at least 6 hours before scanning. PET scans for the $\beta$-amyloid radioligand ${ }^{11} \mathrm{C}$-PiB were acquired using a Philips Allegro PET camera with a resolution of $5.0 \times 5.0 \times 6.5 \mathrm{~mm}^{3}$ $\left(\begin{array}{ll}x & y \\ z\end{array}\right)$. A transmission scan was performed for attenuation correction. PET images were reconstructed using a 3D row action maximum likelihood algorithm with a voxel size of $2.0 \times$ $2.0 \times 2.0 \mathrm{~mm}^{3}(x y z)$. Summed images from the 40- to $70-$ minute time frame were used in each study. Each participant received $370-\mathrm{MBq}{ }^{11} \mathrm{C}-\mathrm{PiB}$ IV over 1 minute. A 30-minute acquisition $(6 \times 5$-minute frames $)$ in a 3D mode starting 40 minutes after injection of ${ }^{11} \mathrm{C}-\mathrm{PiB}$ was performed (total PET scan duration of $1,800 \mathrm{~ms}$ ).

\section{PET processing and analyses in HIV and AlBL data}

All imaging data sets were processed using the PETPVE12 toolbox $^{27}$ for SPM12 (v6906; Wellcome Trust Centre for Neuroimaging, London, UK; fil.ion.ucl.ac.uk/spm) in Matlab 
Table 1 Demographic and clinical characteristics of the study samples

\begin{tabular}{|c|c|c|c|c|}
\hline & HIV+ & AIBL NL & AIBL MCI & AIBL AD \\
\hline $\mathbf{N}$ & 10 & 39 & 7 & 11 \\
\hline Age, y (range) & $55.5(46-68)$ & $68(60-74)$ & $69(64-71)$ & $67(55-74)$ \\
\hline Male (\%) & 100 & 49 & 57 & 55 \\
\hline Education, y (range) & $15(8-19)$ & - & - & - \\
\hline Urban dwelling MSM (\%) & 100 & - & - & - \\
\hline HIV duration, y (range) & $13.8(9.2-29.7)$ & - & - & - \\
\hline Ethnicity (\% white ESB) & 100 & & - & - \\
\hline Current cART duration, mo (range) & $18.5(6-120)$ & - & - & - \\
\hline Nadir CD4 count, cells/mL (range) & $203(11-350)$ & - & - & - \\
\hline Current CD4 count, cells/mL (range) & $488(210-1,008)$ & - & - & - \\
\hline Current CD8 count, cells/mL (range) & $1,074(420-1914)$ & - & - & - \\
\hline Predicted WAIS-III VIQ (range) & $109.6(84.6-121.1)$ & - & - & - \\
\hline GDS (range) & $1.7(0.6-4.7)$ & - & - & - \\
\hline Mini State Mental Examination (range) & - & $30(26-30)$ & $27(24-30)$ & $22(6-28)$ \\
\hline Clinical Dementia Rating (range) & - & $0(0-0.5)$ & 0.5 & $0.5(0.5-3)$ \\
\hline ANI, MND (count) & 4,1 & - & - & - \\
\hline HAD: MSK1, 2, 3 (count) & $1,2,2$ & - & - & - \\
\hline Historical AIDS (\%) & 70 & - & - & - \\
\hline Plasma HIV RNA undetectable ( $<50$ copies $/ \mathrm{mL}$ ) (\%) & 100 & - & - & - \\
\hline CSF HIV RNA undetectable CSF ( $<50$ copies $/ \mathrm{mL}$ ) (\%) & 100 & - & - & - \\
\hline Median CSF A $\beta-42, \mathrm{pg} / \mathrm{mL}$ (range) & $525.2(142.5-663.3)$ & - & - & - \\
\hline Median CSF t-tau, pg/mL (range) & $207.5(73.6-538.5)$ & - & - & - \\
\hline Median CSF p-tau, pg/mL (range) & $61.9(22.6-115.9)$ & - & - & - \\
\hline CSF-AD profile, \% (count) & $43(3 / 7)$ & - & - & - \\
\hline APOE $(\varepsilon 3 / \varepsilon 2) / \varepsilon 4, \%$ (count) & $37.5(3 / 8)$ & $28.2(11 / 39)$ & $28.5(2 / 7)$ & $40(4 / 10)$ \\
\hline APOE $\varepsilon 4 / \varepsilon 4, \%$ (count) & $25(2 / 8)$ & $5.1(2 / 39)$ & $28.5(2 / 7)$ & $40(4 / 10)$ \\
\hline
\end{tabular}

Abbreviations: $\mathrm{AD}=$ Alzheimer disease; $\mathrm{AIBL}=$ Australian Imaging, Biomarkers and Lifestyle; $\mathrm{ANI}=$ asymptomatic neurocognitive impairment; ESB = Englishspeaking background; GDS = global deficit score; HAD = HIV-associated dementia; $\mathrm{MCI}=$ mild cognitive impairment; $\mathrm{MND}=$ mild neurocognitive disorder; $\mathrm{MSM}=$ men who have sex with men; $\mathrm{NL}=$ cognitively normal; VIQ = WAIS-III Verbal IQ.

The AIBL is an Australian cohort that has recruited well-educated participants of white and English-Speaking background similar to that of the HIV cohort (see references 5 and 21 for further details).

Continuous data are presented as median and (range), unless otherwise specified. Percentage values provided for categorical data.

AIDS classification based on the Centers for Disease Control and Prevention (CDC) 1993 HIV disease staging system.

$\mathrm{n}=7 \mathrm{HIV}+$ participants consented to lumbar puncture for CSF analysis.

$\mathrm{n}=8 \mathrm{HIV}+$ participants were genotyped for APOE.

CSF-AD profiles are based on our publication ${ }^{13}$ and were (1) t-tau $>350$ and Aß1-42 <530 pg/mL; (2) p-tau >60 and Aß1-42 <530 pg/mL; and (3) t-tau >350 pg/mL and $A \beta 1-42 / p-t a u<6.5$.

Memorial Sloan Kettering (MSK) scale. ${ }^{24}$ Note that the MSK1 = MND as per Frascati criteria.

r2012b (Mathworks Inc., Sherborn, MA), which has improved functions to handle amyloid PET partial volume effects (PVEs) compared with previous versions. First, all T1weighted images were segmented and skull stripped using default parameters. Second, all PET images were coregistered to their associated T1 images using the toolbox's default parameters. PVE correction was performed using the Müller-
Gärtner method, ${ }^{28}$ and the intensity of the PVE-corrected PET images was normalized to the average intensity of all cerebellar regions (cerebellum standardized uptake value ratio [SUVRc]) from the Automated Anatomical Labeling (AAL) atlas. $^{29}$ The inverse deformation parameters from the segmentation step were used to transform all AAL regions from the Montreal Neurological Institute to native space, separately 
for all participants. Finally, SUVRc values were extracted for each region of the AAL atlas in native space. To reduce the total number of regions of interest (ROIs), the 116 regions from the AAL atlas were arranged based on a previous atlas nomenclature $^{29}$ and averaged out to result in 22 final ROIs. Twenty-six regions related to the cerebellum and the vermis were used as the cerebellum reference. The remaining 90 regions were collapsed into the 22 ROIs. The algorithm used to collapse these regions was developed by M.S.K, neuroanatomist (see supplementary file table e-5, links.lww.com/NXI/A248).

\section{Statistical analyses}

\section{Amyloid uptake and deposition distributions in the HIV+ sample}

Two HIV cases had abnormal ${ }^{11} \mathrm{C}-\mathrm{PiB}$ uptake (figure e-4, links.lww.com/NXI/A248) in which the $\mathrm{PiB}$ uptake was higher in the skull and some parts of the white matter compared with cortical regions. These were subsequently excluded based on the recommendation of the Austin PET team. As a result, $8 \mathrm{HIV}$ cases were considered in the following analyses. The SUVRc data were not normally distributed for any of the study groups. Therefore, all analyses were based on nonparametric statistics.

\section{Group analyses}

Study groups (HIV, NL, MCI, and AD) were compared using the Kruskal-Wallis test followed by the Steel-Dwass method to compare all pairs. There were no covariates entered in the analyses. Although the sex ratio did not significantly differ in the AIBL data $(p>0.50$, and showed a slightly wider variance when women were included, table 2 ), the analyses were repeated excluding only the female participants in the AIBL NL group $(\mathrm{N}=19)$ compared with the HIV sample, to account for a residual effect of sex. Owing to the limited sample size, this was not performed in the MCI (male $=4$ ) and $\mathrm{AD}$ (male $=6$ ) groups. These statistical analyses were conducted using the statistical package JMP 13 (SAS Institute Inc).

\section{Case analyses}

Case 2, on visual inspection, clearly exhibited high amyloid deposition across the entire brain. To quantify how significant the amyloid deposition was in this case, we ran case analysis statistics using the program Singlims_ES.exe $\mathrm{e}^{30-32}$ against the $\mathrm{AD}, \mathrm{MCI}$, and NL groups (AIBL women included).

Case 1 had increased amyloid deposition in the hippocampus on visual inspection. Based on this, a single-case analysis was run for this ROI against the $\mathrm{AD}, \mathrm{MCI}$, and $\mathrm{NL}$ groups. Two other cases (cases 3 and 5) had a noticeable decrease in amyloid deposition across the basal ganglia. To quantify any significant differences, case statistics were run for these 2 cases for the cingulum, caudate, pallidum, putamen, and thalamus against the $\mathrm{AD}, \mathrm{MCI}$, and $\mathrm{NL}$ groups.

\section{Long-term clinical outcomes in the HIV+ sample}

We collected long-term clinical information from medical records located at St. Vincent's Hospital (Sydney, NSW,
Australia) and by contacting the participant's primary physicians. This allowed determination as to which patients had progressive dementia and other relevant comorbid conditions 8-10 years after the PET data collection.

\section{Data availability}

The HIV sample data are available on the UNSW Australia repository: ros.unsw.edu.au/ (search by author "Cysique" and title of the current study). The AIBL data are available upon request to the AIBL group at aibl.csiro.au.

\section{Results}

\section{Group analyses}

The HIV group did not differ statistically from the entire AIBL NL group on any comparisons. The HIV group showed a statistically lower amyloid deposition than the entire MCI group in the caudate and pallidum nuclei. Furthermore, the HIV group obtained a statistically lower amyloid deposition than the entire $\mathrm{AD}$ group in all regions, except for the hippocampi, amygdala, temporal pole, and thalamus. On all ROIs, the $\mathrm{AD}$ groups exhibited higher amyloid deposition than the NL group. The MCI group obtained higher amyloid deposition than the NL group on all regions, except for the thalami, hippocampi, occipital, parietal, and superior temporal regions (table 2 and figure 1 ). The analyses including only male participants produced similar results (table 2). Although because of a slightly more restricted variance in the AIBL NL male subgroup, we found a significantly lower amyloid deposition in the HIV sample in the medial frontal and superior parietal cortex.

\section{Case analyses}

On individual inspection, case 2 (aged 66 years) had high amyloid deposition consistent with $\mathrm{AD}$ (whole-brain $\mathrm{SUVR}_{\mathrm{c}}$ $=2.83$ ) (figures 1 and 2). On quantitative single-case analysis, case 2 showed a significantly increased deposition compared with the NL group, but no difference when compared with the $\mathrm{MCI}$ and $\mathrm{AD}$ cases. When including only men in the analyses, significant differences were again found between case 2 and the $\mathrm{NL}$ and $\mathrm{AD}$ groups, but not compared with the MCI group. These results are compiled in table 3 , and individual ${ }^{11} \mathrm{C}$-PiB PET images are provided (figure 2) and show that case 2 had the highest amyloid deposition compared with all the other groups. As described in table 3, case 2 was APOE $\varepsilon 4$ homozygous with a CSF profile, based on standard cutoff values, consistent with $\mathrm{AD}$. A detailed clinical history and neuropsychological profile for case 2 is presented in table 2. It shows a mix of mild and moderate impairment in cognitive domains affected by HIV-related brain injury (attention/ working memory and speed of information processing), but not for motor functions. The memory profile was consistent with probable $\mathrm{AD}$ with moderate to severe impairment.

Case 1 (aged 62 years) had elevated amyloid deposition in both hippocampi (table e-1, links.lww.com/NXI/A248). Single-case analysis showed that there was no significant 


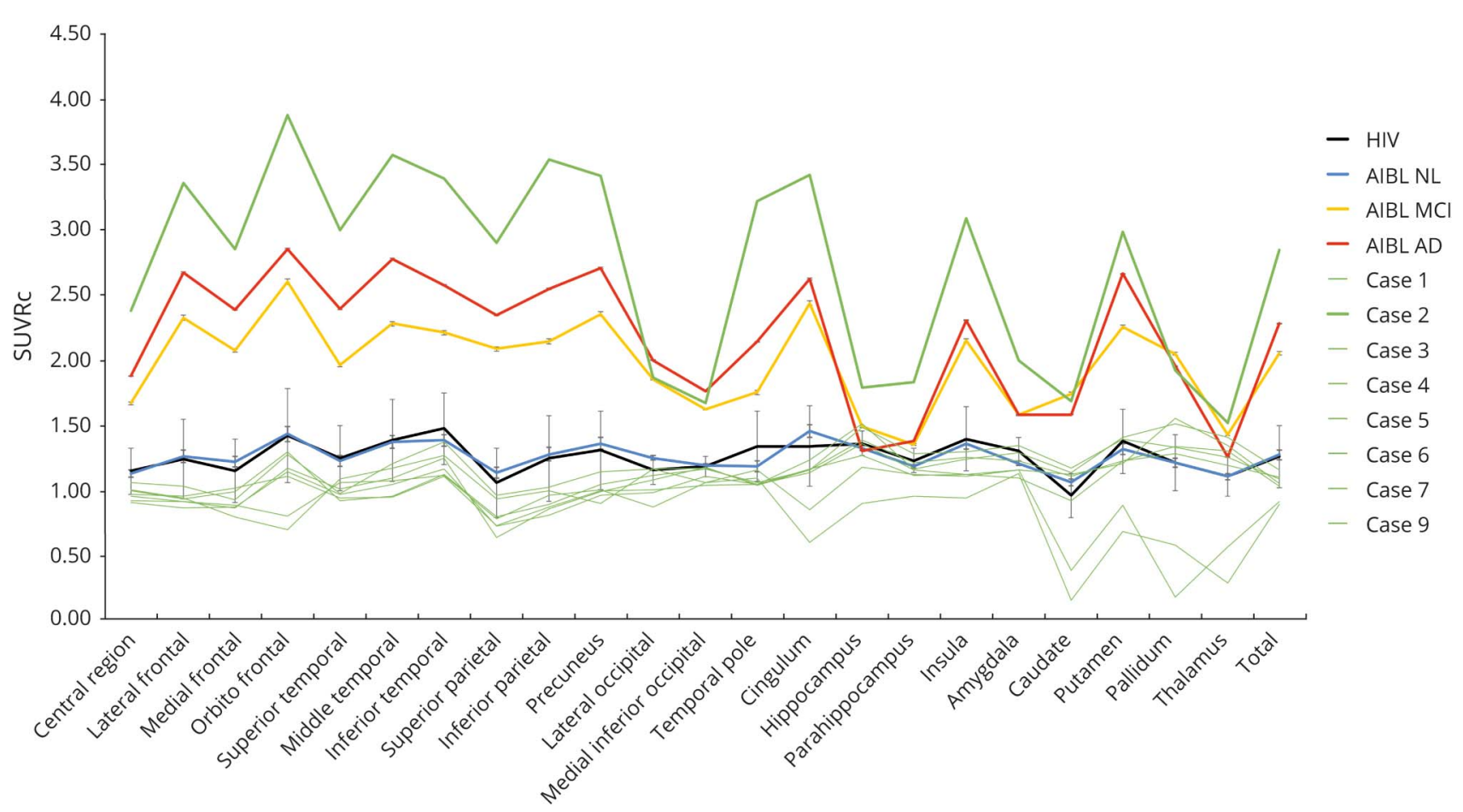

The HIV+ group data are represented by the black line, the AIBL NL group data are represented by the blue line, the AIBL MCI group is represented by the yellow line, and the AIBL AD group is represented by the red line. Mean and SE were provided for all 22 brain regions and total brain SUVRc. Individual HIV data were also represented by the green line, with the case who had amyloid deposition in line with $A D$ in a bolded green line. $A D=A l z h e i m e r$ disease; $A I B L=$ Australian Imaging, Biomarkers and Lifestyle; HAND = HIV-associated neurocognitive disorder; $\mathrm{MCI}=\mathrm{mild}$ cognitive impairment; NL = cognitively normal; $\mathrm{ROI}=$ region of interest; SUVRC = standardized uptake value ratio with reference to the cerebellum.

increase in deposition compared with the $\mathrm{MCI}$ and $\mathrm{AD}$ groups, but there was a trend compared with the NL group $(p=0.06$; table 2 and figure 2). This individual was also APOE $\varepsilon 4$ homozygous; however, CSF biomarkers were not consistent with an $\mathrm{AD}$ profile $(\mathrm{CSF} \mathrm{A} \beta-42=168.21$ $\mathrm{pg} / \mathrm{mL}$; $\mathrm{t}$-tau $=132.18 \mathrm{pg} / \mathrm{mL}$; and $\mathrm{p}$-tau $=36.23 \mathrm{pg} / \mathrm{mL})$. Neuropsychological deficits included mildly impaired speed of information processing and working memory and moderately impaired motor functions with preserved verbal fluency and memory.

Case analysis statistics, as well as demographic, neuropsychological, HIV, CSF, and APOE data for case 3 (aged 52 years) and case 5 (aged 54 years) are presented in table e-2, links.lww.com/NXI/A248, and e-3, links.lww.com/ $\mathrm{NXI} / \mathrm{A} 248$, respectively, and in figure 2 . There was a significant decrease in amyloid deposition for both cases in all areas tested (range $p<0.04-p<0.0001$ ), except for the cingulum and putamen when compared with the NL group $(p=0.15-0.07)$. Furthermore, case 5 was identified as having an $\mathrm{AD}$-like CSF profile. Both were cases with severe $H A D$.

\section{Long-term follow-up}

The 2 patients who originally had clinically severe $\mathrm{HAD}$ (MSK3) and 1 who had clinically moderate HAD (MSK2) and had normal amyloid died because of other comorbidities (see table e-4, links.lww.com/NXI/A248). Case 2 who had clinically moderate $\mathrm{HAD}+\mathrm{AD}$ (MSK2) and significantly elevated amyloid also died after progression to severe dementia. Case 1 who had ANI, with elevated amyloid in the hippocampi, developed a progressive supranuclear palsy (PSP)-like illness, which has remained static. Case 1 also showed microinfarcts and hyperintensities of the white matter consistent with vascular injury on MRI and evidence of cognitive decline reaching an MND (MSK1) diagnosis within 3 years after study. Other cases, which had normal amyloid, included 1 ANI case (case 9), who did not progress clinically according to their physicians and are currently living independently. Case 6, who had MND (MSK1) at entry, has been cognitively stable despite a series of cancers, which have been successfully treated. Case 7, who had clinically mild HAD (MSK1) at entry, has been mostly stable except for progressing peripheral neuropathy. Of the 10 cases, 2 cases (cases 8 and 10) showed abnormal PiB PET uptake (figure e-4, links.lww.com/NXI/A248). Case 8 had ANI at entry that progressed clinically displaying worsening mood changes and behavioral problems with subsequent cognitive decline consistent with MND (MSK1). Case 10 also progressed clinically by reporting increasing cognitive difficulties in line with MND (MSK1). These 2 cases have not reached the HAD stage and still live independently. 
Table 2 Comparisons of amyloid deposition on the study groups

\begin{tabular}{|c|c|c|c|c|c|c|c|c|}
\hline ROI & HIV+ & AIBL NL & AIBL NL male & AIBL MCI & AIBL AD & $p 1$ & $p 2$ & $\begin{array}{l}p<0.05 \text { on } \\
\text { Steel-Dwass }\end{array}$ \\
\hline Total & $1.04(0.12)$ & $1.08(0.22)$ & $1.08(0.13)$ & $2.17(0.70)$ & $2.40(0.43)$ & $<0.0001$ & 0.37 & $d, e, f$ \\
\hline Central region & $0.95(0.07)$ & $1.01(0.16)$ & $1.00(0.14)$ & $1.78(0.84)$ & $1.91(0.37)$ & $<0.0001$ & 0.22 & $d, f$ \\
\hline Lateral frontal & $0.92(0.04)$ & $0.99(0.30)$ & $1.00(0.17)$ & $2.44(0.86)$ & $2.73(0.42)$ & $<0.0001$ & 0.11 & $d, e, f$ \\
\hline Medial frontal & $0.87(0.09)$ & $1.02(0.29)$ & $1.03(0.31)$ & $2.25(0.79)$ & $2.49(0.35)$ & $<0.0001$ & $<0.05$ & $d, e, f$ \\
\hline Orbitofrontal & $1.13(0.27)$ & $1.14(0.21)$ & $1.13(0.20)$ & $2.71(0.53)$ & $2.92(0.53)$ & $<0.0001$ & 0.87 & $d, e, f$ \\
\hline Superior temporal & $0.96(0.08)$ & $1.02(0.17)$ & $1.03(0.16)$ & $2.19(0.91)$ & $2.36(0.68)$ & $<0.0001$ & 0.46 & $d, f$ \\
\hline Middle temporal & $1.05(0.13)$ & $1.11(1.16)$ & $1.10(0.15)$ & $2.64(1.04)$ & $2.84(0.72)$ & $<0.0001$ & 0.20 & $d, e, f$ \\
\hline Inferior temporal & $1.15(0.14)$ & $1.18(0.21)$ & $1.19(0.19)$ & $2.36(0.82)$ & $2.51(0.64)$ & $<0.0001$ & 0.87 & $d, e, f$ \\
\hline Superior parietal & $0.77(0.14)$ & $0.91(0.30)$ & $0.99(0.23)$ & $2.36(1.65)$ & $2.32(0.52)$ & 0.0001 & 0.04 & $d, f$ \\
\hline Inferior parietal & $0.87(0.11)$ & $1.02(0.26)$ & $1.04(0.12)$ & $2.62(1.34)$ & $2.62(0.54)$ & $<0.0001$ & 0.02 & $d, f$ \\
\hline Precuneus & $0.98(0.05)$ & $1.10(0.28)$ & $1.12(0.20)$ & $2.66(1.16)$ & $2.69(0.49)$ & $<0.0001$ & 0.10 & $d, e, f$ \\
\hline Lateral occipital & $1.07(0.15)$ & $1.12(0.28)$ & $1.07(0.19)$ & $1.95(0.86)$ & $1.88(0.60)$ & $<0.0001$ & 0.15 & $d, f$ \\
\hline Medial inferior occipital & $1.09(0.11)$ & $1.11(0.16)$ & $1.11(0.21)$ & $1.55(0.63)$ & $1.65(0.39)$ & $<0.0001$ & 1.0 & $d, f$ \\
\hline Temporal pole & $1.03(0.03)$ & $1.05(0.14)$ & $1.06(0.18)$ & $1.69(0.45)$ & $2.24(0.71)$ & $<0.0001$ & 0.79 & $e, f$ \\
\hline Cingulum & $1.14(0.17)$ & $1.22(0.21)$ & $1.23(0.19)$ & $2.42(0.78)$ & $2.71(0.27)$ & $<0.0001$ & 0.15 & $d, e, f$ \\
\hline Hippocampi & $1.34(0.21)$ & $1.32(0.14)$ & $1.32(0.16)$ & $1.42(0.24)$ & $1.31(0.25)$ & 0.07 & 0.63 & $f$ \\
\hline Parahippocampi & $1.14(0.07)$ & $1.14(0.10)$ & $1.13(0.09)$ & $1.25(0.23)$ & $1.36(0.20)$ & 0.0008 & 0.67 & $e, f$ \\
\hline Insula & $1.10(0.13)$ & $1.18(0.26)$ & $1.16(0.16)$ & $2.14(0.38)$ & $2.28(0.62)$ & $<0.0001$ & 0.91 & $d, e, f$ \\
\hline Amygdala & $1.14(0.11)$ & $1.15(0.09)$ & $1.15(0.08)$ & $1.50(0.25)$ & $1.65(0.40)$ & $<0.0001$ & 0.67 & $e, f$ \\
\hline Caudate & $1.10(0.48)$ & $0.99(0.30)$ & $0.95(0.33)$ & $1.66(0.53)$ & $1.37(0.59)$ & $<0.0001$ & 0.59 & $b, d, e, f$ \\
\hline Putamen & $1.21(0.26)$ & $1.19(0.25)$ & $1.15(0.15)$ & $2.33(0.40)$ & $2.56(0.46)$ & $<0.0001$ & 0.42 & $d, e, f$ \\
\hline Pallidum & $1.31(0.49)$ & $1.15(0.33)$ & $1.13(0.28)$ & $2.02(0.25)$ & $2.07(0.73)$ & $<0.0001$ & 0.25 & $b, d, e, f$ \\
\hline Thalamus & $1.24(0.44)$ & $1.11(0.23)$ & $1.11(0.25)$ & $1.35(0.34)$ & $1.22(0.37)$ & 0.03 & 0.25 & $f$ \\
\hline
\end{tabular}

Abbreviations: $\mathrm{AD}=$ Alzheimer disease; $\mathrm{AIBL}=$ Australian Imaging, Biomarkers and Lifestyle; $\mathrm{MCl}=$ mild cognitive impairment; $\mathrm{NL}=$ cognitively normal; $\mathrm{ROI}=$ region of interest; SUVRc = standardized uptake value ratio with reference to the cerebellum.

SUVRc values presented as median (IQR).

$p 1$ is the overall $p$ value extracted from the Kruskal-Wallis tests.

$p 2$ is the Wilcoxon $p$ value for the comparison between the HIV sample and male NL from AIBL.

$p<0.05$ on the all pairs, Steel-Dwass method multiple comparisons: (a) NL vs HIV; (b) MCI vs HIV; (c) MCI vs AD; (d) HIV vs AD; (e) NL vs MCl; and (f) NL vs AD; ns = not significant at $p<0.05$ on all comparisons.

\section{Discussion}

Consistent with previous studies, ${ }^{7-9}$ there was no statistical difference in amyloid deposition between the HIV group and NL cases (combining men and women) from the AIBL. However, the NL cases, as part of the AIBL study, were older than those in previous referenced studies. When repeating the analyses with only NL male participants from the AIBL, we found abnormally low amyloid deposition in the HIV group in the medial frontal and superior parietal cortex. This indicates that HIV may be associated with abnormally low amyloid deposition when compared with an older NL cohort. Our study has a small HIV sample size, yet it was a data-rich sample composed of well-characterized HIV+ cases aged 55.5 (46-68) years old, with formally diagnosed HAND, longterm HIV disease clinical stability, viral suppression, and low neurologic and psychiatric confounds. Critically, the sample had long-term follow-up of the participants over years (table e-4, links.lww.com/NXI/A248). This is unique compared with previous HIV PET studies. The small HIV sample size was amenable to a case analysis strategy, which showed abnormal increases of amyloid deposition in 2 HAND cases.

Case 2 had an overall deposition of amyloid consistent with $\mathrm{AD}$ supported on single-case analysis. The typical AD-like CSF profile and progressive cognitive impairment with a mixed 
Table 3 Case 2 with a probable AD and MND neuropsychological profile concomitant to the PET examination and clinical history

\section{Demographic and clinical data}

Age, $y$

Education, $y$

66

HIV duration, $y$

18

\section{Current cART duration, mo}

Nadir CD4 count, cells/mL

Current CD4 count, cells/mL

Current CD8 count, cells/mL

24.6

Predicted WAIS-III VIQ

21

GDS

Historical AIDS

CSF $A \beta-42$

CSF t-tau

21

CSF p-tau

18

CSF-AD-like profile

APOE

210

882

121.1

1.25

Yes

142.5

328

71.7

Yes

$\varepsilon 4 / \varepsilon 4$

Total SUVRc vs NL

$<0.0001(3.60)(2.77-4.52)^{a}$

Total SUVRc vs MCI

0.11 (1.31) (0.30-2.45)

Total SUVRc vs AD

Total SUVRc vs NL (males only)

$0.07(1.60)(0.72-2.60)$

Total SUVRc vs MCl (males only)

$0.002(3.30)(2.12-4.57)^{a}$

Total SUVRc vs AD (males only)

$0.10(1.58)(0.08-3.40)$

$0.04(2.18)(0.71-3.96)^{a}$

\section{Neuropsychological data}

Cognitive domains

Degree of cognitive impairment

Attention/working

Mild impairment

Speed of information processing

Mild impairment

Mental flexibility

Mild to moderate impairment

Verbal learning

Mild to moderate impairment

Verbal active retrieval

Verbal memory retention

Moderate to severe impairment

Verbal fluency

\section{Motor}

Moderate to severe impairment

\section{-}

Abbreviations: $\mathrm{AD}=$ = Alzheimer disease; GDS = global deficit score; $\mathrm{HAND}=\mathrm{HIV}$-associated neurocognitive disorder; $\mathrm{MCl}=$ mild cognitive impairment; $\mathrm{MND}$ = mild neurocognitive disorder; NL = cognitively normal; SUVRc = cerebellum standardized uptake value ratio; VIQ = WAIS-III Verbal IQ.

Case statistics reported as 1 -tailed $p$ value (t value) (confidence interval).

The reported neuropsychological data were normed against local normative data. ${ }^{22}$

This case has been in the clinical care of Sydney St. Vincent's Hospital HIV clinics for more than 25 years. His cognitive decline was documented across 18 years through standard neurologic (CSF, blood, and MRI/MRS examinations) and neuropsychological examinations at the neurology department. In 2001, this case was diagnosed with mild HAND in which symptoms remained stable for the next 10 years (mild learning and mild mental flexibility impairment). His premorbid abilities were in the superior range. His progressive memory decline started 8-9 years after his initial HAND diagnosis ( $\sim 1$ year ahead of the PET) as shown in the table above. At this time, he showed no apraxia, no agnosia, and no reasoning deficits (data from a standard clinical neuropsychological assessment performed during this period), in addition to no language and no motor deficits for the research examination as shown in the table. It is not until the PET examination that AD was suspected. Within 2-3 years, he progressed to severe dementia; this started with a decline in attention/working memory, speed processing, and further learning/memory decline. He lived for 6 years at this stage.

${ }^{a} p<0.05$. 


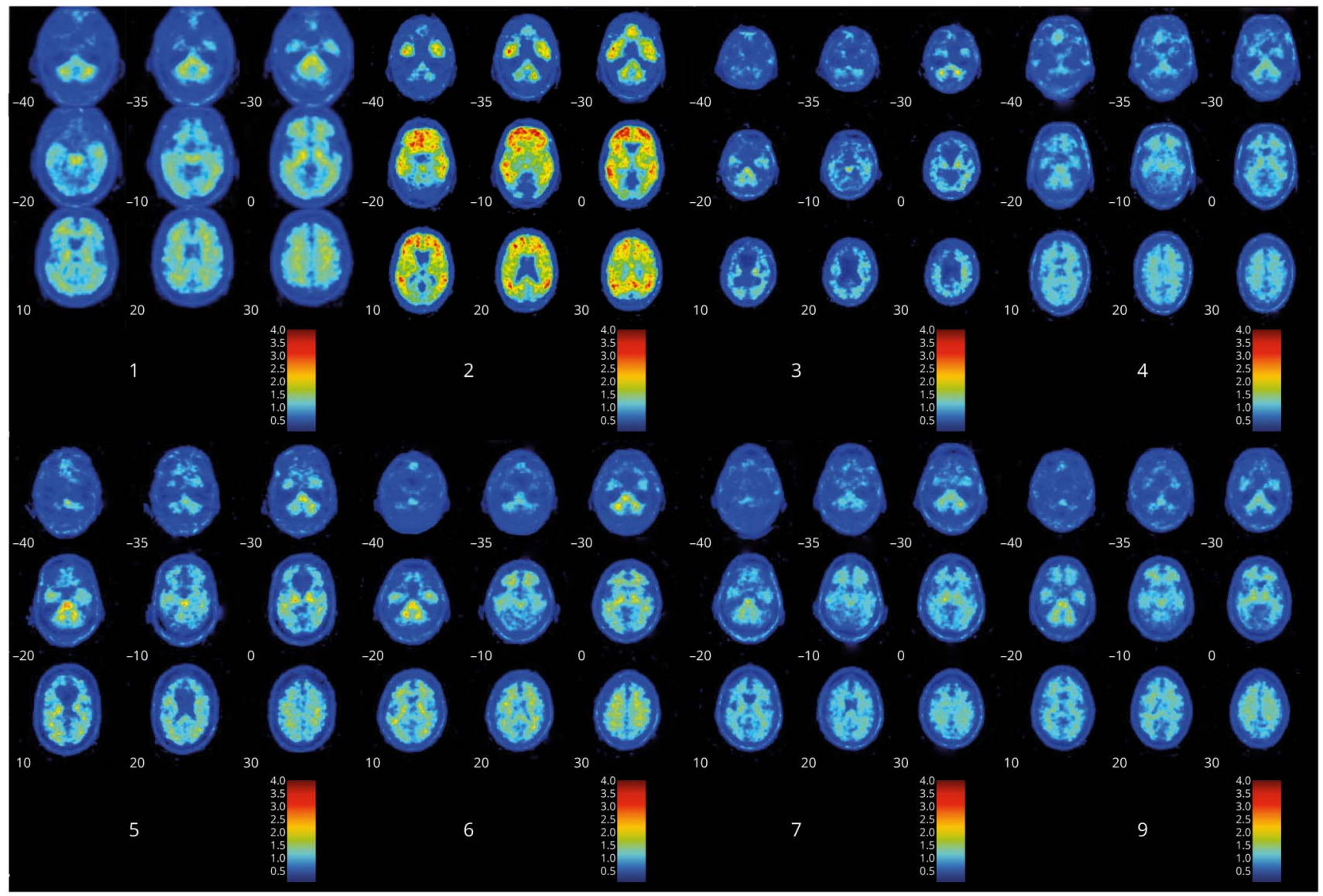

Raw PiB PET data are presented in the 8 HIV cases. Case 2 is the case with high amyloid deposition. Case 1 is the case with a trend for higher hippocampi binding compared with the Australian Imaging, Biomarkers and Lifestyle NL group. Cases 3 and 5 were the cases with the lowest amyloid deposition across all groups. Note that case example for the NL, MCl, and Alzheimer disease groups is presented in supplemental material in figures e-1, e-2, and e-3, respectively, links.Iww.com/NXI/A248. MCI = mild cognitive impairment; $\mathrm{NL}=$ cognitively normal.

picture of typical $\mathrm{AD}$ and HAND deficits supported a probable dominant $\mathrm{AD}$ diagnosis. It is important that case 2 was $\mathrm{APOE}$ E4 homozygous and was the second oldest participant in the sample (66 years of age). This is consistent with potential coexistence of HAND and AD in an advanced HIV+ patient, as previously described. ${ }^{10}$ From an $\mathrm{AD}$ prevalence perspective, it is logical that some HIV+ patients will develop $\mathrm{AD}$ based on genetic and other predisposing factors. It is, however, not clear whether the start of neurodegeneration may be earlier because of HIV. It is also uncertain whether amyloid deposition may have a faster deposition rate, ${ }^{33}$ although this is certainly possible given that this case had the highest amount of amyloid deposition across all our comparisons. Also, we noted that the cognitive profile was unusual compared with HAND (sparing of motor functions and long-term stability of psychomotor speed) and $\mathrm{AD}$ profiles (restricted memory deficits with no agnosia, apraxia, or language deficits except toward the end stage).$^{34}$ From a clinical perspective, it has been argued that HAND and $\mathrm{AD}$ can be distinguished to some degree. ${ }^{2}$ However, an extensive test battery with the inclusion of agnosia, apraxia, and language would be needed in addition to the usual cognitive domains assessed in HAND to better address this question. It has been noted that ${ }^{11} \mathrm{C}-\mathrm{PiB}$ PET, which has high affinity and selectivity for fibrillar $A \beta$ in plaques and extracellular amyloid deposits, ${ }^{5}$ may lead to lower binding in PLHIV and patients with HAND for whom plaques have been found to be more often diffuse and intracellular. ${ }^{16-18}$ In this study, case 2 shows that such an interpretation may not be correct in aging PLHIV with a possible coincidental $\mathrm{AD}$ pathology and profile.

A role for HIV in the dysregulation of brain amyloid is biologically plausible. Hypotheses include recent research into the antimicrobial activity of amyloid ${ }^{35,36}$ in which increased amyloid may reflect a long-term response to the presence of HIV infection in the brain (potentially in brain cellular reservoirs). Supporting this, Bourgade et al. ${ }^{37,38}$ suggest that $\beta$-amyloid can inhibit herpes simplex virus 1 and is associated with overproduction of amyloid more generally in infectionrelated neurologic diseases.

Although case 1 showed no statistical overall increase in amyloid deposition, a trend toward abnormal binding in the hippocampus was observed. The CSF biomarker profile was also not consistent with an $\mathrm{AD}$-like profile. Recent literature on $\mathrm{AD}$ 
suggests that, even subthreshold increases in amyloid levels can result in memory deficits, and in some cases it is the rate of amyloid accumulation, rather than the overall amyloid level, that more accurately predicts worsening memory. ${ }^{39,40}$ However, case 1 had a PSP-like illness, the pathologic underpinning of which in the context of HIV is unknown at present. Nonetheless, it is interesting that he was homozygous for APOE E4. There is evidence that progression into a PSP-like illness is associated with a homozygous APOE E4 genotype. ${ }^{41}$

In this study, 2 of the 3 cases (cases 3 and 5) with severe HAD (pre-cART) who survived on cART showed low amyloid deposition. These cases were the most vulnerable and died within 8-10 years of the study's long-term outcomes collection. All were younger than 65 years with long HIV duration ( $>25$ years). Cases 3 and 5 exhibited major subcortical atrophy, consistent with severe HIV-related brain injury. ${ }^{42-45}$ This level of subcortical atrophy potentially biased the measurement of amyloid in the basal ganglia. Nevertheless, there is at least 1 plausible mechanism for the abnormally low level of amyloid. Microglia and astrocytes primed by HIV may play important roles in engulfing and purging $A \beta$ plaques from the brain. ${ }^{46}$ In severe $\mathrm{HAD}$ with a disrupted blood-brain barrier, amyloid clearance may be enhanced in some individuals. Also, in treated PLHIV, amyloid clearance may occur through HIV-1 Tat priming and activation of microglia. ${ }^{47}$ Case 5 had a CSF biomarker profile that supported $\mathrm{AD}$ (T-tau $>350 \mathrm{Ab} 42 / \mathrm{P}$-tau $<6.5$ ), but the PET scan did not confirm increased amyloid deposition suggesting caution in the clinical use of CSF for $\mathrm{AD}$ diagnosis in patients with HIV, especially those with HAND. Finally, we cannot exclude that in these younger cases, the plaques may have been diffuse rather than fibrillar, and this may have contributed to a lower ${ }^{11} \mathrm{C}-\mathrm{PiB}$ PET signal. ${ }^{16-18}$

Although our study does not provide statistical evidence of an overall increase in amyloid in PLHIV with HAND, it highlights the importance of interindividual variations in patients with chronic HIV and HAND and the need to focus the research on favorable and unfavorable phenotypic and/or biotypic clusters. $^{48}$ In this small sample, we were able to detect cases with high, focused (albeit trending) and very low brain amyloid. Such complex phenotypes if already apparent in a pilot study suggest a need to focus on long-term individual phenotypic analyses. Future longitudinal studies monitoring amyloid deposition as PLHIV age are crucial in understanding the relationship between abnormal amyloid levels and HAND as well as other comorbidities. It is noteworthy that all the HIV+ patients in this study also had a large number of medical comorbidities (See table e-4). How exactly such comorbidities played a role in their amyloid pattern and then clinical progression or lack thereof remains to be fully elucidated. Although we used a PET protocol that retained the individual neuroanatomy, it would be important that larger studies use a multimodal imaging strategy because abnormal brain aging in HIV can be detected by using volumetric MRI. ${ }^{49}$
Our study had some limitations. The controls used in this study were obtained from AIBL and included a mix of male and female participants differing from the HIV+ sample who were all men. The sex difference had a minimal effect on the cases with $\mathrm{AD}+\mathrm{HAD}$, when specifically investigated. We also had missing data for the APOE genotyping and CSF profiles and recognize this, just as for previous studies have, as a limitation. Although 50\% of the HAND cases had HAD as per Frascati criteria at study entry, it is important to note that the MSK clinical staging was well represented as well as those with mild HAND as per Frascati criteria. Finally, our pilot sample size and case series analyses yield indicative rather than definitive findings. It remains unclear why 2 HAND cases had no cortical amyloid uptake. Despite a thorough search, we could not find reports of similar issues in the HIV or non-HIV literature. There was no PET scan technical issue reported by the Austin Health PET Centre examiners.

In conclusion, relative to NL older controls, brain amyloid burden does not differ in virally suppressed HAND at the group level. However, individual analyses show that abnormally high and low amyloid burden occur. These results indicate that focus on individual phenotypes in longitudinal analyses in the HIV-aging cohort is needed.

\section{Acknowledgment}

The authors thank the participants for their time on the study and dedicate this work to the patients whom they cared for and who have since died. They thank the physicians of the HIV+ patients for responding in a timely and detailed fashion to their queries. They thank Dr. Jones, Prof. Villemagne, and Prof. Rowe from the Centre for PET, Austin Health, Melbourne, Australia, for their support in interpreting the data acquisition validity.

\section{Study funding}

This work was supported by the NHMRC project grants (APP568746; PI Cysique, APP1105808; PI Brew, and APP1045400; PI Cysique), Peter Duncan Neuroscience Research Unit at St. Vincent's Hospital Centre for Applied Medical Research (Director: Prof. Brew), and a UNSW Australia Faculty of Medicine development.

\section{Disclosure}

B.J. Brew reports personal fees from $\mathrm{AbbVie}$, personal fees from $\mathrm{ViiV}$, grants and personal fees from Biogen Idec, personal fees from Merck Sharp and Dohme, grants from the National Health and Medical Research Council, and grants from the National Institutes of Health, outside the submitted work. C.D. Rae reports personal fees from Philips Healthcare, personal fees from Springer, and personal fees from Elsevier, outside the submitted work. Other authors report no declaration of interest. Go to Neurology.org/NN for full disclosures.

\section{Publication history}

Received by Neurology: Neuroimmunology \& Neuroinflammation October 7, 2019. Accepted in final form April 6, 2020. 
Appendix Authors

\begin{tabular}{|c|c|c|}
\hline Name & Location & Contributions \\
\hline $\begin{array}{l}\text { Gemma } \\
\text { Howdle, } \\
\text { BA\&Sc }\end{array}$ & NeuRA/UNSW & $\begin{array}{l}\text { Analysis of data and } \\
\text { manuscript writing }\end{array}$ \\
\hline $\begin{array}{l}\text { Yann Quidé, } \\
\text { PhD }\end{array}$ & NeuRA/UNSW & $\begin{array}{l}\text { Analysis of data and } \\
\text { manuscript writing }\end{array}$ \\
\hline $\begin{array}{l}\text { Mustafa S. } \\
\text { Kaseem, PhD }\end{array}$ & NeuRA & Manuscript writing \\
\hline $\begin{array}{l}\text { Kate Johnson, } \\
\text { MD }\end{array}$ & $\begin{array}{l}\text { St. Vincent's Hospital } \\
\text { Research Campus }\end{array}$ & $\begin{array}{l}\text { Acquisition and analysis of } \\
\text { data and manuscript writing }\end{array}$ \\
\hline $\begin{array}{l}\text { Caroline D. } \\
\text { Rae, PhD }\end{array}$ & NeuRA/UNSW & Manuscript writing \\
\hline $\begin{array}{l}\text { Bruce J. Brew, } \\
\text { MD, DSc, } \\
\text { FRACP, FAAN }\end{array}$ & $\begin{array}{l}\text { St. Vincent's Hospital } \\
\text { Research Campus, } \\
\text { UNSW }\end{array}$ & $\begin{array}{l}\text { Conception and design; } \\
\text { acquisition and analysis of } \\
\text { data; and manuscript writing }\end{array}$ \\
\hline $\begin{array}{l}\text { Lucette A. } \\
\text { Cysique, PhD }\end{array}$ & $\begin{array}{l}\text { NeuRA/UNSW, St. } \\
\text { Vincent's Hospital } \\
\text { Research Campus }\end{array}$ & $\begin{array}{l}\text { Conception and design; } \\
\text { acquisition and analysis of } \\
\text { data; and manuscript writing }\end{array}$ \\
\hline
\end{tabular}

\section{References}

1. Mackiewicz MM, Overk C, Achim CL, Masliah E. Pathogenesis of age-related HIV neurodegeneration. J Neurovirol 2019;21:019-00728.

2. Milanini B, Valcour V. Differentiating HIV-associated neurocognitive disorders from Alzheimer's disease: an emerging issue in geriatric neuroHIV. Curr HIV/AIDS Rep 2017;14:123-132.

3. Edmonds EC, Bangen KJ, Delano-Wood L, et al. Patterns of cortical and subcortical amyloid burden across stages of preclinical Alzheimer's disease. J Int Neuropsychol Soc 2016;22:978-990.

4. Sarro L, Senjem ML, Lundt ES, et al. Amyloid- $\beta$ deposition and regional grey matter atrophy rates in dementia with Lewy bodies. Brain 2016;139:2740-2750.

5. Villemagne VL, Burnham S, Bourgeat P, et al. Amyloid beta deposition, neurodegeneration, and cognitive decline in sporadic Alzheimer's disease: a prospective cohort study. Lancet Neurol 2013;12:357-367.

6. Achim CL, Barrio JR, Kepe V, et al. Abnormal protein aggregation in the brains of long-term surviving HIV patients in the HAART era. 8th International Symposium on NeuroVirology: Journal of Neurovirology 2007:12.

7. Ances BM, Christensen JJ, Teshome M, et al. Cognitively unimpaired HIV-positive subjects do not have increased 11C-PiB: a case-control study. Neurology 2010;75:111-115.

8. Ances BM, Benzinger TL, Christensen JJ, et al. 11C-PiB imaging of human immunodeficiency virus-associated neurocognitive disorder. Arch Neurol 2012;69:72-77.

9. Ortega M, Ances BM. Role of HIV in amyloid metabolism. J Neuroimmune Pharmacol 2014;9:483-491.

10. Turner RS, Chadwick M, Horton WA, Simon GL, Jiang X, Esposito G. An individual with human immunodeficiency virus, dementia, and central nervous system amyloid deposition. Alzheimers Dement (Amst) 2016;4:1-5

11. Soontornniyomkij V, Moore DJ, Gouaux B, Soontornniyomkij B, Sinsheimer JS, Levine AJ. Associations of regional amyloid-beta plaque and phospho-tau pathology with biological factors and neuropsychological functioning among HIV-infected adults. J Neurovirol 2019;29:019-00761.

12. Chang L, Jiang C, Cunningham E, et al. Effects of APOE $\varepsilon 4$, age, and HIV on glial metabolites and cognitive deficits. Neurology 2014;82:2213.

13. Cysique LA, Hewitt T, Croitoru-Lamoury J, et al. APOE epsilon 4 moderates abnormal CSF-abeta-42 levels, while neurocognitive impairment is associated with abnormal CSF tau levels in HIV+ individuals-a cross-sectional observational study. BMC Neurol 2015;15:51.

14. Geffin R, McCarthy M. Aging and apolipoprotein E in HIV infection. J Neurovirol 2018;24:529-548.

15. Mishra S, Blazey TM, Holtzman DM, et al. Longitudinal brain imaging in preclinical Alzheimer disease: impact of APOE epsilon4 genotype. Brain 2018;141:1828-1839.

16. Rempel HC, Pulliam L. HIV-1 tat inhibits neprilysin and elevates amyloid beta. AIDS 2005; 19:127-135.

17. Green DA, Masliah E, Vinters HV, Beizai P, Moore DJ, Achim CL. Brain deposition of betaamyloid is a common pathologic feature in HIV positive patients. AIDS 2005;19:407-411.

18. Umlauf A, Soontornniyomkij B, Sundermann EE, et al. Risk of developing cerebral beta-amyloid plaques with posttranslational modification among HIV-infected adults. AIDS 2019;33:2157-2166.
19. Achim CL, Adame A, Dumaop W, Everall IP, Masliah E; Neurobehavioral Research Center. Increased accumulation of intraneuronal amyloid beta in HIV-infected patients. J Neuroimmune Pharmacol 2009;4:190-199.

20. Cysique LA, Jugé L, Gates T, et al. Covertly active and progressing neurochemical abnormalities in suppressed HIV infection. Neurol Neuroimmunol Neuroinflamm 2018;5:e430. 10.1212/NXI.0000000000000430.

21. Ellis KA, Bush AI, Darby D, et al. The Australian Imaging, Biomarkers and Lifestyle (AIBL) Study of Aging: methodology and baseline characteristics of 1112 individuals recruited for a longitudinal study of Alzheimer's disease. Int Psychogeriatr 2009;21:672-687.

22. Cysique LA, Heaton RK, Kamminga J, et al. HIV-associated neurocognitive disorder in Australia: a case of a high-functioning and optimally treated cohort and implications for international neuroHIV research. J Neurovirol 2014;20:258-268.

23. Cysique LA, Moffat K, Moore DM, et al. HIV, vascular and aging injuries in the brain of clinically stable HIV-infected adults: a (1)H MRS study. PLoS One 2013;8:e61738.

24. Price RW, Brew BJ. The AIDS dementia complex. J Infect Dis 1988;158:1079-1083.

25. Rowe CC, Ng S, Ackermann U, et al. Imaging $\beta$-amyloid burden in aging and dementia. Neurology 2007;68:1718.

26. The Kirby Institute. HIV, Viral Hepatitis and Sexually Transmissible Infections in Australia Annual Surveillance Report 2018: Sydney: UNSW Australia, 2018.

27. Gonzalez-Escamilla G, Lange C, Teipel S, Buchert R, Grothe MJ; Alzheimer's Disease Neuroimaging Initiative. PETPVE12: an SPM toolbox for partial volume effects correction in brain PET-Application to amyloid imaging with AV45-PET. Neuroimage 2017;147:669-677.

28. Muller-Gartner HW, Links JM, Prince JL, et al. Measurement of radiotracer concentration in brain gray matter using positron emission tomography: MRI-based correction for partial volume effects. J Cereb Blood Flow Metab 1992;12:571-583.

29. Tzourio-Mazoyer N, Landeau B, Papathanassiou D, et al. Automated anatomical labeling of activations in SPM using a macroscopic anatomical parcellation of the MNI MRI single-subject brain. Neuroimage 2002;15:273-289.

30. Crawford JR, Garthwaite PH. Investigation of the single case in neuropsychology: confidence limits on the abnormality of test scores and test score differences. Neuropsychologia 2002;40:1196-1208.

31. Crawford JR, Garthwaite PH, Porter S. Point and interval estimates of effect sizes for the case-controls design in neuropsychology: rationale, methods, implementations, and proposed reporting standards. Cogn Neuropsychol 2010;27:245-260.

32. Crawford JR, Howell DC. Comparing an individual's test score against norms derived from small samples. Clin Neuropsychologist 1998;12:482-486.

33. Fulop T, Witkowski JM, Larbi A, Khalil A, Herbein G, Frost EH. Does HIV infection contribute to increased beta-amyloid synthesis and plaque formation leading to neurodegeneration and Alzheimer's disease? J Neurovirol 2019;25:637-647.

34. Rubin LH, Sundermann EE, Moore DJ. The current understanding of overlap between characteristics of HIV-associated neurocognitive disorders and Alzheimer's disease. J Neurovirol 2019;25:661-672.

35. Brothers HM, Gosztyla ML, Robinson SR. The physiological roles of amyloid- $\beta$ peptide hint at New ways to treat Alzheimer's disease. Front Aging Neurosci 2018;10:118.

36. Moir RD, Lathe R, Tanzi RE. The antimicrobial protection hypothesis of Alzheimer's disease. Alzheimer's Dement 2018;14:1602-1614.

37. Bourgade K, Garneau H, Giroux G, et al. beta-Amyloid peptides display protective activity against the human Alzheimer's disease-associated herpes simplex virus-1. Biogerontology 2015;16:85-98.

38. Bourgade K, Le Page A, Bocti C, et al. Protective effect of amyloid-beta peptides against herpes simplex virus- 1 infection in a neuronal cell culture model. J Alzheimers Dis 2016;50:1227-1241.

39. Leal SL, Lockhart SN, Maass A, Bell RK, Jagust WJ. Subthreshold amyloid predicts tau deposition in aging. J Neurosci 2018;38:4482-4489.

40. Landau SM, Horng A, Jagust WJ; Alzheimer's Disease Neuroimaging Initiative. Memory decline accompanies subthreshold amyloid accumulation. Neurology 2018;90:e1452-e1460.

41. Tsuboi Y, Josephs KA, Cookson N, Dickson DW. APOE E4 is a determinant for Alzheimer type pathology in progressive supranuclear palsy. Neurology 2003;60:240-245.

42. Tesic T, Boban J, Bjelan M, Todorovic A, Kozic D, Brkic S. Basal ganglia shrinkage without remarkable hippocampal atrophy in chronic aviremic HIV-positive patients. J Neurovirol 2018;24:478-487.

43. Sanford R, Fernandez Cruz AL, Scott SC, et al. Regionally specific brain volumetric and cortical thickness changes in HIV-infected patients in the HAART era. J Acquir Immune Defic Syndr 2017;74:563-570.

44. Aylward EH, Henderer JD, McArthur JC, et al. Reduced basal ganglia volume in HIV-1associated dementia: results from quantitative neuroimaging. Neurology 1993;43:2099-2104.

45. Becker JT, Sanders J, Madsen SK, et al. Subcortical brain atrophy persists even in HAART-regulated HIV disease. Brain Imaging Behav 2011;5:77-85.

46. Li JW, Zong Y, Cao XP, Tan L, Tan L. Microglial priming in Alzheimer's disease. Ann Transl Med 2018;6:176.

47. Chivero ET, Guo ML, Periyasamy P, Liao K, Callen SE, Buch S. HIV-1 Tat primes and activates microglial NLRP3 inflammasome-mediated neuroinflammation. J Neurosci 2017;37:3599-3609.

48. Paul R. Neurocognitive phenotyping of HIV in the era of antiretroviral therapy. Curr HIV/AIDS Rep 2019;16:230-235.

49. Chang L, Shukla DK. Chapter 18 - imaging studies of the HIV-infected brain. In: Brew BJ, editor. Handbook of Clinical Neurology. Amsterdam, The Netherlands: Elsevier; 2018:229-264. 


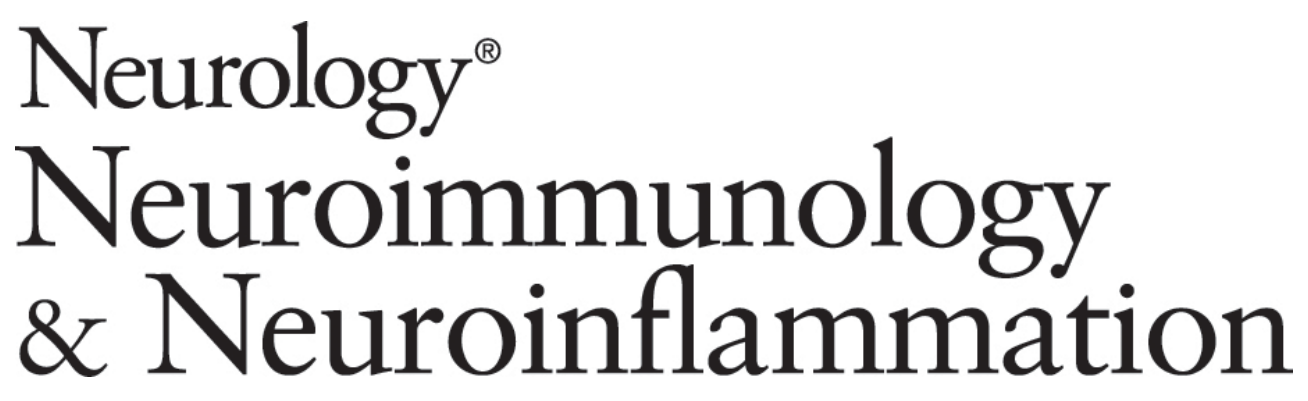

Brain amyloid in virally suppressed HIV-associated neurocognitive disorder Gemma C. Howdle, Yann Quidé, Mustafa S. Kassem, et al.

Neurol Neuroimmunol Neuroinflamm 2020;7;

DOI 10.1212/NXI.0000000000000739

This information is current as of May 11, 2020

Neurol Neuroimmunol Neuroinflamm is an official journal of the American Academy of Neurology.

Published since April 2014, it is an open-access, online-only, continuous publication journal. Copyright

Copyright $\odot 2020$ The Author(s). Published by Wolters Kluwer Health, Inc. on behalf of the American

Academy of Neurology.. All rights reserved. Online ISSN: 2332-7812.

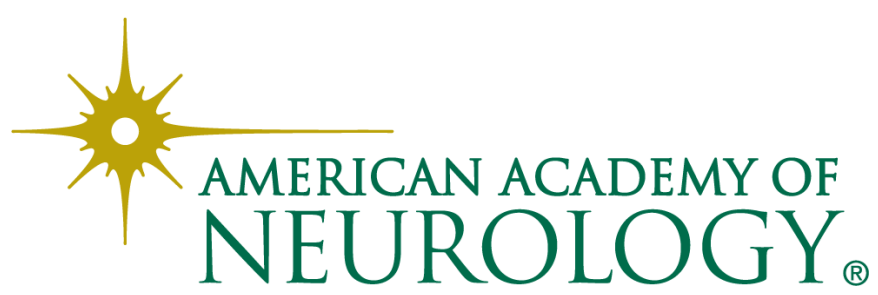




\section{Updated Information \& Services}

References

Subspecialty Collections

Permissions \& Licensing

Reprints including high resolution figures, can be found at: http://nn.neurology.org/content/7/4/e739.full.html

This article cites 46 articles, 3 of which you can access for free at: http://nn.neurology.org/content/7/4/e739.full.html\#\#ref-list-1

This article, along with others on similar topics, appears in the following collection(s):

Alzheimer's disease

http://nn.neurology.org//cgi/collection/alzheimers_disease

HIV dementia

http://nn.neurology.org//cgi/collection/hiv_dementia

MCI (mild cognitive impairment)

http://nn.neurology.org//cgi/collection/mci_mild_cognitive_impairmen $\mathrm{t}$

Neuropsychological assessment

http://nn.neurology.org//cgi/collection/neuropsychological_assessment PET

http://nn.neurology.org//cgi/collection/pet

Information about reproducing this article in parts (figures,tables) or in its entirety can be found online at:

http://nn.neurology.org/misc/about.xhtml\#permissions

Information about ordering reprints can be found online:

http://nn.neurology.org/misc/addir.xhtml\#reprintsus

Neurol Neuroimmunol Neuroinflamm is an official journal of the American Academy of Neurology.

Published since April 2014, it is an open-access, online-only, continuous publication journal. Copyright

Copyright $\odot 2020$ The Author(s). Published by Wolters Kluwer Health, Inc. on behalf of the American Academy of Neurology.. All rights reserved. Online ISSN: 2332-7812.

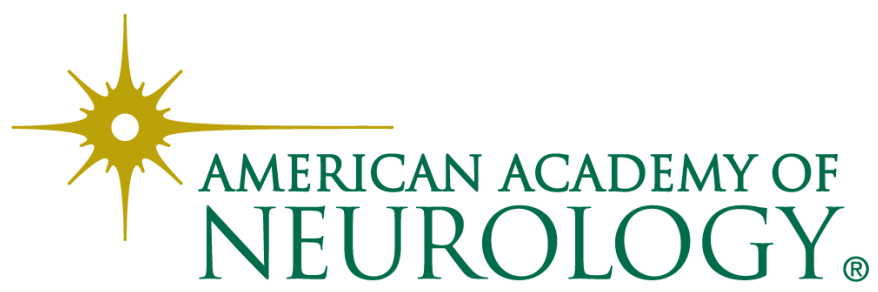

